The following alphabetical and geographical lists represent NALLD membership through September 30, 1981. Please send corrections directly to:

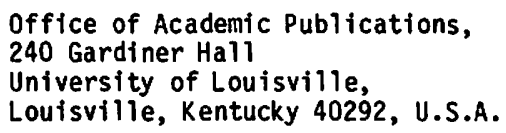

This copyrighted membership list is the property of the National Association of Learning Laboratory Directors (NALLD) and may not be used for commercial purposes without specific permission of NALLD. For information concerning the use of this list, contact the Editor of the NALLD Journal/Technology and Mediated Instruction:

\author{
Dale V. Lally, Jr., Director \\ Arts \& Sciences Learning Laboratory \\ 16 Bingham Humanities Building \\ University of Louisville \\ Louisville, Kentucky 40292, U.S.A.
}

\title{
ALPHABETICAL LIST
}

Abed, A. Z., Foreign Language Laboratory, Louisiana State University, Baton Rouge, LA 70803

Abrams, W., Education Department, Capital Complex, Carson City, NV 89710

Acadia University, Vaughn Memorial Library, Wolfville, NS BOP IXO CANADA

Achiba, Machiko, 8-34 Komu inshukusha, 11-72 Sakashita, Okazaki, Aichi-ken 444 JAPAN

ADFL BULLETIN, Editor, 62 Fifth Avenue, New York, NY 10011

Aikens, H. F., Part-Time Studies \& Ext, Dalhousie University, Halifax NS B3H 3J5 CANADA

Allen, Ernest, 1 Alien Farm Road, Fort Worth, TX 76116

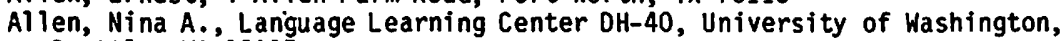
Seattle, WA 98195

Allen, Wendy W., Language Laboratory, Saint 01 af College, Northfield, MN 55057

Al tman, Howard B., Dept. of Modern Languages, University of Louisvilie, Louisville, KY 40292

Alyta, Kenneth J., International Programs Office, Stevens Institute of Technology, Hoboken, NJ 07030

Amano, Kazuo, 16-6 Shiomigaoka-Cho, Chiba-Shi 280 JAPAN

Annamalai, E., Central Inst. Indian Lang, Manasagangotri, Mysore-570 006 INDIA

Appalachian State University, Serials Department, Belk Library, Boone, NC 28608

App 1 Linguistics Inst. Belgium, Attn: Willy Martin, Blijde Inkomststraat 21, 3000 Leuven BELGIUM

Arizona State University, Library-Periodicals, Tempe, AZ 85281

Arnold, E. P., Dept. of Languages, Clemson University, Clemson, SC 29631

Arnold, Robert L., Instructional Resources, University of Central Florida, Box 25,000 , Orlando, FL 32816

Association for Educational Communications \& Technology, Attn: Charles Van Horn, 1126 Sixteenth St. NW, Washington, D.C. 20036 
Aucoin, G. E., 34 Langley Avenue, Dartmouth NS B2W 2Y6 CANADA

Augerot, James E., Language Learning Center, University of Washington, Seattle, WA 98195

Aulestia, Victor H., Language Media Center, University of Maryland, 5401 Wilkins Avenue, Baltimore, MD 21228

AV LANGUAGE JOURNAL, Attn: Editor, University of Aston-Birmingham, Gosta Green, Birmingham B4 7ET ENGLAND

AV LANGUAGE JOURNAL, 7 Shelley Close, Langley, Bucks SL3 8JW ENGLAND

Babin, J. W., French, St. Francis Xavier University, Antigonish, NS B2G 1 CO CANADA

Bailey, Leslie F., Language Laboratories-48B Kresge, Northwestern University, 1859 Sheridan Rd., Evanston, IL 60201

Baker, R., Foreign Language Supervisor, Education Department, 65 South Front St., Columbus, $\mathrm{OH} 43215$

Ball State University, Dept. of Library Service, Muncie, IN 47306

Baltra, Liliana, CPEIP, Casilla 16162, Santiago 9 CHILE

Barnes, J., Department of Education, Agana GUAM 96910

Barr, Charles J., Language Laboratory, St. John's Seminary College, 5118 East Seminary Road, Camarililo, CA 93010

Bartz, W., Public Instruction, State House 229, Indianapolis, IN 46204

Bator, Joseph W., Language Development, Pan American World Airways, Kennedy International Airport, Jamaica, NY 11430

Bauman, Ronald A., Modern European Languages, Pomoma College, Claremont, CA 91711

Beaudoin, John R., Language Laboratory, Western Michigan University, Kalamazoo, MI 49008

Bennet, T.J.A., Language Laboratories, Hochschule St. Gallen, Dufourstrasse 50, St. Gallen, 9014 SWITZERLAND

Berndt, Robert J, Dept of Foreign Language \#4140, SW Missouri State University, Springfield, MO 65802

Berry, Kenneth Russel1, Media Services, Santa Barbara City College, 721 Cliff Drive, Santa Barbara, CA 93109

Beusch, A., Department of Education, P. 0. Box 8717, BWI Airport, Baltinore, MD 21240

Bocaz-Moraga, Sergio, Dept. of Modern Languages, Eastern Washington University, 3144 Patterson Hail, Cheney, WA 99004

Bosworth, Lewis A., New Student Services, University of Wisconsin, 432 N. Murray St., Madison, WI 53706

Boudreau, Jacqueline, Language Lab., Wheaton College, Norton, MA 02766

Bowen, Louise H., 201 Bartlett Hall, Dartmouth College, Hanover, NH 03755

Bowie State College, Pullen Library-Periodicals, Bowie, MD 20715

Boyden, Patrick C., Self-Instruction Center, Kent State University, 265 Library, Kent, OH 44242

Bridgers, W. K., Learning Laboratories, University of California, Phelps Hall, Santa Barbara, CA 93106

Brinton, Donna, English IESL, UCLA, 405 Hilgard Ave., Los Angeles, CA 90024

British Library, Accessions, Lending Div., Boston Spa, Wetherby, Yorkshire LS23 $7 B Q$ ENGLAND

Bronx Community College, Library-Learning Center, West 181st Street, Bronx, NY 10453

Brown, C. P. Foreign Language Department, Western Kentucky University, Ivan Wilson Building 251, Bowling Green, KY 42101

Buchandlung Lehmkuhl, Leopolds trasse 45,8000 Munchen 40 WEST GERMANY

Burggraaf, Samuel E., Learning Res. Ctr., Brighan Young University, W-162 Stadium, Provo, UT 84602

Butler, Samuel R., 1095 South Downing Street, Denver, CO 80209

Caldwell, Wm. A., Language Laboratory, The American University, Massachusetts \& Nebraska Aves. N.W., Washington, D.C. 20016 
California Polytech State University, Library-Periodicals, San Luis Obispo, CA 93407

Calvin College Library, 3207 Burton, S.E., Grand Rapids, MI 49506

Capretz, Pierre J., Language Laboratory, Yale University, 111 Grove Street, New Haven, CT 06510

Carleton College, Library, Northfield, MN 55057

Carlson, Florence, AV Director, Wellesley College, Wellesley, MA 02181

Carter, Thomas P., Learning Resource Services, Daihousie University, Killam Library, Halifax, NS B3H 4 H8 CANADA

Cassidy, Maureen, Foreign Languages, Dept. of Education, 1270 Queen Emma St, Rm. 1201, Honolulu, HI 96813

Cate, Robert L., Modern Language Laboratory, University of Texas-EI Paso, El Paso, TX 79968

Cate, Bob, P.0. Box 260133, Tampa, FL 33685

Chang, Gerald K. J., Foreign Language Laboratory, University of Hawaii, 1890 East-West Road, Honolulu, HI 96822

Cheatham, Rosalie M., Language Laboratory, University of Arkansas-Little Rock, 6405 Evergreen Rd., Little Rock, AR 72207

Cherry, Charles Maurice, Modern Language Laboratory, Furman University, Greenville, SC 29613

Chestnut, D., Education Department, Education Building, Box 911, Harrisburg, PA 17126

Ciceran, A. J., Language Laboratory, Brock University-Decew Campus, St. Catharines, Ont L2S 3Al CANADA

Clark, D. J., Language Lab, Gilman Hall, Johns Hopkins University, Baltimore, MD 21218

Cleveland State University, Library-Serials, $1860 \mathrm{E}$. 22nd Street, Cleveland, $\mathrm{OH}$ 44115

Clifford, Billy B., 5353 Arlington Expressway, Apt. 9N, Jacksonville, FL 32211

Cochran, $\mathrm{Cl}$ ay, 205 North Walnut Street, Brazii, IN 47934

Coffey, Thomas F., Classics \& Modern Languages, Creighton University, Omaha, NE 68178

College of Cape Breton, Library, Sydney Campus, Box 5300, Sydney, NS B1P 6L2 CANADA

Coltharp, Jerry, A/N Service, 340 Gaskill Hall, Miami University, Oxford, $O H$ 45056

Comet, Honore, Language Lab-Suite 500, All-World Language Institute, 280 South Beverly Drive, Beverly Hills, CA 90212

Concordia University, Library, Serials Acquisitions, Sir George Williams Campus, 1455 Maisonneuve Blvd., West, Montreal PQ H3G 1M8 CANADA

Conner, Maurice W., Central States Conference, University of Nebraska, P.0. Box 688 , Omaha, NE 68101

Conweli, Marilyn J., 251 W. Dekalb Pike, Apt 909C, King of Prussia, PA 19406

Cormier, Ramona, $149 \mathrm{Bal}$ dwin, Bowling Green, $\mathrm{OH} 43402$

Couroux, Gerard, College Marie Victorin, 7000 rue Marie Victorin, Montreal, $P Q$ 462 CANADA

Cox, Ronald M., Engl ish Program-Personnel, Kobe Steel, Ltd., 3-18, 1-chome, Wakinohama-cho, Chuo-ku, Kobe 651 JAPAN

Cox, Shirley 0., Language Laboratory, Auburn University, Auburn, AL 36830

Criminale, Leonard R., Language Laboratory, Elmira College, Elmira, NY 14901

Crosbie, K., Bilinguai Instruction, Public Instruction, 01d Capitol Building, Olympia, WA 98504

Ctr Info Lang Teaching, Deputy Librarian, 20 Carlton House Terrace, London SW iY 5AP ENGLAND

Dalstrom, E. Kay, Foreign Languages, University of Nebraska, Omaha, NE 68112

Dammer, P., Foreign Language Education, Education Department, Albany, NY 12234

David Yellin Teacher's Sem, Beth-Hakerem, Jerusalem ISRAEL 
Davies, Norman, University of Linkoping, S-581 83 Linkoping SWEDEN

De Cerchio, Donna Ellis, Foreign Language Department, West Chester State College, West Chester, PA 19380

de Johnson, Teresa, Dept. of Modern Languages, St. Louis University, St. Louis, MO 63103

de la Portilla, Marta, Spanish Department, Manhattanville College, Purchase Street, Purchase, NY 10577

DeMers, Irene, Foreign Languages, Harvard School, 3700 Coldwater Canyon Road, North Hollywood, CA 91604

Department of Education, Director, Bilingual Education, Boston, MA 02111

Devargas, Dolores, Instituto de Idiomas, Carilla 51, Tarija BOLIVIA

D. H. Hi1l Library, Periodicals Service Center, Box 5007-NC State, Raleigh, NC 27650

DLIWC ACademic Library, DLIFLC-Building 618, Presidio, M/F DAKF 03-80-M-3315, Monterey, CA 93940

Dodge, James W., Secretary Treasurer, Northeast Conference, P. 0. B0x 623, Middl ebury, VT 05753

Dowling, J. K., Language Laboratory, University of Queensland, St. Lucia, Brisbane 4067 AUSTRALIA

Dowling, J. K., Language Laboratory, University of Queensland, St. Lucia, Brisbane 4067 AUSTRALIA

Dugan, Marjorie, Learning Laboratory, Community College-Philadelphia, 34 South 11 th Street, Philadelphia, PA 19107

DuVerlie, Claude, Modern Languages \& Linguistics, UMBC, Catonsville, MD 21228

Earle, Betty B., 59 Henderson Street, Needham, MA 02192

Eastern Montana College, Library-Serials Dept., Billings, MT 59101

Eaton, Shirley, 204 West Monument Avenue, Hatboro, PA 19040

Edmonds, Victor, Media Services, Our Lady of the Lake University, 411 SW 24 th Street, San Antonio, TX 78285

Educational Electronics-California, Attn: Bernard Keach, 213 North Cedar Avenue, Ingl ewood, CA 90301

Educational Media Division, Attn: R. Pohlmeier, P.0. Box 20604, Oklahoma City, OK 73156

Educational Media Division, Attn: Jim Goodin, P.0. Box 20604, OKlahoma City, OK 73156

Educational Media Division, Attn: Shelby Hardage, P.0. Box 20604, Oklahoma City, OK 73156

Ellison, Keith, Department of Foreign Languages, West Georgia College, Caroliton, GA 30118

Elmendorf, William E., Language Laboratory, Western Washington State College, Bellingham, WA 98225

Enos, Jacqueline, Foreign Language Dept., Bridgewater State College, Bridgewater, MA 02324

Entin, Nathaniel A., Gratz College, 10th Street \& Tabor Road, Philadelphia, PA 19141

ERIC Exchange, LR, Center for Applied Linguistics, 3520 Prospect Street, NW, Washington, D.C. 20007

Feagin, C., Department of Education, Education Annex-Trinity, Atlanta, GA 30303

Fisher, Ronald D., Language Laboratory, LaSalle College, 20 th Street at 01 ney Avenue, Philadelphia, PA 19141

Florida State University, Serials, Strozier Library, Tallahassee, FL 32306

Ford, Kathleen, Instructional Laboratory, UCLA, 362 Royce Ha11, Los Angeles, CA 90024

Ford, Penelope, Instructional Media Resources, Language Ctr./Univ. of Maryland, Catonsville, MD 21228

Forquer, Mary Beth, Learning Center, Lehigh University-Coppee Hall 3, Bethlehem, PA 18015 
Forth, Joli, 973 Kingston Avenue, Piedmont, CA 94611

Fournier, R., Bilingual Education, 64 North Main Street, Third Floor, Concord, NH 03301

Francis, Larry P., Intensive English Program, University of Missouri, 228 Gentry, Columbia, MO 65201

Frank, Stephania E., University Hall, Bowling Green State University, Bowling Green, $\mathrm{OH} 43402$

Frechette, Ernest A., Foreign Language Education, Florida State University, Tallahassee, FL 32306

Free University of Berlin, Foreign Language Laboratories, Habelschwerdter Allee 45, Innenstrasse 24,1 Berlin 33 (Dahlem) HEST GERMANY

Freudenstein, Reinhold, Foreign Language Research Info. Ctr., Lahnberge, D-3550 Marburg/Lahn WEST GERMANY

Fritsch, Hans J., P. 0. Box 61, Whately, MA 01093

Froehlich, Jurgen, Language Laboratory, Scripps College, Claremont, CA 91711

Frost, Arthur F., Language Laboratory, Princeton University, 304 E. Pyne Building, Princeton, NJ 08540

Fukuoka University, LL Room, Nanakuma, Fukuoka 814 (MZ) JAPAN

Galloway, V., Foreign Language Consultant, Education Department, 801 Rutledge, Columbia SC 29201

Garinger, Ermal E., Language Laboratories, University of Kansas, 4069 Wescoe Hail, Lawrence, KS 66045

Gemmer, Bill, Kamehameha Schools, Keona-Lower Campus, Honolulu, HI 96817

Gennaula, Maryedna Gillespie, 5872 Kings School Road, Bethel Park, PA 15102

GERMAN QUARTERLY, Editor, German \& Slavic Languages, University of Virginia, Charlottesvilie, VA 22901

Gifu Jyoshi Tanki Daigaku, 2693 Fukumi tsu, Nagara, Gifu 502 MZ JAPAN

Gilde, Hans M., University of Nebraska, OIdfather 1111, Lincoln, NE 68588

Gillespie, Netta, Language Laboratory, University of Illinois, G-89 Foreign Languages, Urbana, IL 61801

Gilmore, Roger H., Foreign Languages, Colorado State University, Fort Collins, CO 80523

Gionet, Arthur J., Foreign Lang \& Lit, North Texas State Univ, Denton, TX 76203

Goldsmith, Stanley, Language Laboratory, Columbia University, 101-B Philosophy Hall, New York, NY 10027

Goldsworthy, Thomas, P.0. Box 537, Odessa, FL 33556

Gore, Peter B., P. O. Box 11, Deerfield, MA 01342

Goulet, Sr. Noella, Notre Dame College, 2321 Elm Street, Manchester, NH 03104

Governors State University, Library/Serials-Kardex, Park Forest South, IL 60466

Griffith, P., Education, 100 North First Street, Springfield, IL 62777

Grittner, F., Foreign Language Education, Public Instruction, 126 Langdon, Madison, WI 53702

Gwinn, Joei A., Physics Department, University of Louisville, Louisville, KY 40292

Hal1, Bunelle M., Monterey Inst. Int'1. Studies, 425 Van Buren Street, Monterey, CÁ 93940

Hamm, C., Language Laboratories, Queen's University, Kingston, Ont K7L 3N6 CANADÁ

Hamnerly, Hector, Literature \& Linguistics, Simon Fraser University, Burnaby, BC V5A IS6 CANADA

Hanzel, Louis F., Nanzan University, 18 Yamazato-cho, Showa-ku, Nagoya 466 JAPAN

Hartwell, Jerry, Instructional Media Center, Bowling Green State University, Bowling Green, $\mathrm{OH} 43403$

Harvey, Edward, Hawail Campus, Brigham Young University, Box 33 BYU-HC, Laie, HI 96762 
Heather, Anthony J., Language Resource Center, Loyola University, Chicago, IL 60626

Heilenman, Laura K., Dept. of French \& Italian, Northwestern University, Evanston, IL 60201

Heinle \& Heinle Publishers, Inc., Attn: Charles Heinle, 51 Sleeper Street, Boston, MA 02210

Helsingen Yliopisto, Kielikeskus, Fabianinkatu 26, SF-00100 Helsinki 10 FINLAND

Hennig, C. W., Language Laboratory, University of Waterloo, Room 110, ML Building, Waterioo, Ont N2L 3G1 CANADA

Herrera, Lazaro M., Language Laboratory, Westminster Schools, 1424 West Paces Ferry, N.W., Atianta, GA 30327

Hewson, J., Linguistics, Memorial University of Newfoundland, St. John's, Nfind CANADA

Hi11, Ann, Language Laboratory, Randolph-Macon Woman's College, 2400 Rivermont Avenue, Lynchburg, VA 24503

HISPANIA, Attn: Albert Turner, Glenbrook South High School, 4000 West Lake Avenue, Glenview, IL 60025

HISPANIA, Editor, Romance Languages, University of Cincinnati, Cincinnati, OH 45221

Hilts, Margarete, La Sierra College, Loma Linda University, Riverside, CA 92515

Hiroshima Daigaku, Honbu Toshokan-K, Higashisendamachi, Hiroshima 730 MZ JAPAN

Hiroshima Shudo Daigaku Lib, Otsuka Numata-Cho, Hiroshima (Kin) 731-31 JAPAN

Hirsch, Bernice G., Foreign Language Laboratory, Samford University, 800 Lakeshore Drive, Bi rmingham, AL 35229

Hoff, Roma, 335 Bartlett Court, Eau Claire, WI 54701

Honda, Ritsuri, Assumption College, 2-16 Ote-cho, Takatsuki-shi, 0saka 569 JAPAN

Howard, J., Department of Education, 111 Coliseum Boulevard, Montgomery, AL 36109

Howard University, Language Lab/903301, Room 361 Locke Hall, 2441 6th Street, N.W., Washington, D.C. 20059

Howard University Library, Acquisitions Dept., Serials, Room \#105, Washington, D.C. 20059

Howe, E., Foreign Language Education, Education Department, 250 East 500 South, Salt Lake City, UT 84111

Hudson, G., Department of Education, Little Rock, AR 72201

Hughett, Harvey, Foreign Languages \& Literatures, University of Idaho, Moscow, ID 83843

Ikeura, Sadahiko, Fukuoka University of Education, 279 Akama Munakata-gun, Fukuoka JAPAN

Infante, J., Languages, Public Instruction Department, Raleigh, NC 27611

Instructional Materials Center, 201 East Nine Mile Road, Highland Springs, VA 23075

Inter-American University, Library, San German, PR 00753

Irving, Evelyn Uhrhan, 2508 Glen Elm Drive, N.E., Cedar Rapids, IA 52402

Jabalquinto, Sergio Rios, Casilla 165, Tarija BOLIVIA

Jackson, D., Second Languages, Public Instruction, Helena MT 59601

Jackson, Gordon, 257 Cliffiew Drive, Gahanna, OH 43230

James, E. F., Language Lab, Rm.259B, University College-Univ. of Toronto, 15 King's College Circle, Toronto, Ont. M5S 1A1 CANADA

Jebe, S., Department of Education, 649 Capitol Square, 550 Cedar Street, St. Paul, MN 55101

Jewish National \& University Library, Periodicals, P.0. Box 503, Jerusalem ISRAEL

Johnson, Barbara, Foreign Language Department, Washington State University, Pullman, WA 99164 
Jahnson, Tom, Audio-Visual Marketing, TELEX COMMUNICATIONS, INC., 9600 Aldrich Avenue South, Minneapolis, MN 55420

Jordan, Terence C., Language Centre, Griffith University, Nathan, Queensland 4111 AUSTRALIA

Jyvaskyla University, Language Laboratory, SF-40/100 Jyvaskyla 10 FINLAND

Kagawa University, Library, Saiwai-Cho, Takamatsu-Shi 760 (Kin) JAPAN

Kanagawa University, Library, Rokkakubashi, Kanagawa-Ku, Yokohama (MZ) 221 JAPAN

Kansai University Library, P. 0. Box 50, Suita, Osaka 564 MZ JAPAN

Kennedy, Dora F., Foreign Language Department, Prince Georges' Public School, 4806 Harvard Road, College Park, MD 20740

Kenner, R., Language Laboratory-A/N Dept., Concordia University H-399, 1455 de Maisonneuve Blvd., Montreal, PQ H3G IM8 CANADA

Kessler, Michael, Lang Lab-Humanities, San Francisco State Univ, 1600 Holloway, San Francisco, CA 94132

King, R., Education Department, P. 0. Box 480, Jefferson City, M0 65102

Kipphorn, Jr., Richard A., Language Laboratory, Saint Joseph's University, Philadelphia, PA 19131

Kirk, David, 124 Chace Street, Santa Cruz, CA 95060

Kitao, Kenji, Dept. of English, Doshisha University, Karilgyo-ku, , Kyoto 602 JAPAN

Kodama, Paul, Learning Assistance Center, Hawaii Pacific College, 1164 Bishop Street, Honolulu, HI 96813

Kogyo Kure Koto, Senmon Gakko Lib, Aga-Minami-2-2-11, Kure 737 MZ JAPAN

Kohmoto, Sutesaburo, 4-16-17 Meguro, Meguro-ku, Tokyo 153 JAPAN

Konyvtar, Egyetemi, POB 483, Budapest 1372 HUNGARY

Korkeakoulun, Joensuun, Kirjasto, PL111, 80101 Joensuu 10 FINLAND

Kozoriz, G. , Canadian Forces Lang School-0tt., National Defense Headquarters, Ottawa, Ont K1A OK2 CANADA

Krupatkin, Yakov Borisovich, u.1. Odesskaja 1, kv. 19, Sevastopol 335000 USSR

Kruse, Jr., E. L., 733 Kirkwood Drive, Grand Is land, NY 14072

Kump, Richard J., 1822 Ricardo Drive, Cape Girardeau, M0 63701

Kurita, Katsumi, SONY Language Laboratory, Hiyama B1dg, \#2, 4F1, 2-20-30, Takanawa, Minato-Ku, Tokyo 108 JAPAN

Kuroda, Takashi, English Literature, Otsuma Womens College, 12, 3-bancho, Chiyoda-ku, Tokyo 102 JAPAN

Kyoto Sangyo Daigaku, Toshokan, (Kyoyo-Eigo-Ken), Kita-Ku Kyoto 603 MZ JAPAN

Kyushu Sangyo Daigaku, (LL Room), 327, Matugadai-2-chome, Higashi-ku, Fukuoka City 813 MZ JAPAN

Lacher, S., Languages, Public Instruction, Capitol Building, Bismarck, ND 58505

Lally, Kenneth, 3222 Murray Hill Pike, Louisville, KY 40222

Lance, John, Japan-Amer Conversation Inst., Intl. Education Center, 21 Yotsuya, 1-chome, Shinjuku-17, Tokyo 160 JAPAN

Lange, Dale L., 130B Peik Hall, University of Minnesota, Minneapolis, MN 55455

LANGUAGE \& LANGUAGE BEHAVIOR ABSTRACTS, P.0. Box 22206, San Diego, CA 92122

Language Instruction Supervisor, Bilingual Education, P. 0. Box 2219, Hartford, CT 06115

Language Instruction Supervisor, Education Department, P. 0. Box 630, St. Thomas, VI 00801

Language Lab. Lib., Brown University, Box E, Providence, RI 02912

Laroche, J., Languages, D. C. Public Schools, 415 12th Street, N.W., Washington, D.C. 20004

LaVallee-Williams, Marthe, 1326 Spruce Street, No. 3001, Philadelphia, PA 19107

Lawrason, Robin E., Media Learning Center, Temple University, Humanities B1dg. HL-21, Philadelphia, PA 19122

Lemyze, J.-C., Language Laboratory, MCGill University, Montreal, PQ H3A IX9 CANADA 
Leonhardt-Hendriks, Helga M., Foreign Language Instructor, 640 San Elijo Street, San Diego, CA 92106

Levinson, Bernice, Dept. of Modern Languages, Brooklyn College-CUNY, Brooklyn, NY 11210

Lewis, Steven F., GEP Training, Lockheed Aircraft International, A.G., Riyadh SAUDI ARABIA

LHTEC, 905 Lindsey Hopkins Library, 1410 N.E. 2nd Avenue, Miami, FL 33132

Library of Congress, Order Division, Continuations Unit, Washington, D.C. 20540

LoRe, A. G., Romance Languages, University of North Carolina, 323 Dey Hall, Chape1 Hill, NC 27614

Long Beach City College, Attn: Lorraine Yocky, Foreign Language Dept., 4901 East Carson Street, Long Beach, CA 90808

Lopez, Jose A., ESPOL, Apartado 5863, Guayaquil 303733 ECUADOR

Loud, Mary, Foreign Languages, Youngstown State University, Youngstown, $\mathrm{OH}$ 44555

Luckau, Paul F., Germanic Languages, Brigham Young University, 270 MSRD, Provo, UT 84602

Macinko, John, Language Laboratories, University of Colorado, 302 01d Main Building, Boulder, CO 80309

Magil1, Robert, Language Lab, Modern Languages, Towson State University, Baltimore, MD 21204

Majhanovich, Suzanne E., Faculty of Education, University of Windsor, Windsor, Ont, N9B 3P4 CANADA

Mandel son, Leonore R., 10 Tobey Brook, Pittsford, NY 14534

Marietta College, Modern Language Department, Marietta, OH 45760

Marino, Anna, Language Laboratory, Hunter College, 695 Park Avenue, Box 375, New York, NY 10021

Marxheimer, Edward, Romance Languages, University of Alberta, Edmonton, A1 ta T6G 2E6 CANADA

Mason, Richard G., Humanities Dept., Michigan Technological University, Houghton, MI 49931

Maynes, J., Department of Education, 1535 West Jefferson, Phoenix, AZ 85007

Mazzeo, Guido E., Romance Languages \& Literatures, George Washington University, 2115 G Street, N.W., Rm. 411, Washington, D.C. 20052

McAndrew, Alex, Language Study Center, University of New South Wales, Sydney, NSW 2006 AUSTRALIA

McCarty, Joan, Language Laboratory, 106 Calkins, Hofstra University, 106 Calkins Hal1, Hemp stead, NY 11550

Mc'Connel1, Richard, Dept. of Foreign Languages, Memphis State University, Memphis, TN 38152

McCune, Jerry, Language Laboratory, Indiana University, Ballantine Hall.120, Bloomington, IN 47401

McGill University Libraries, Serials Department, 3459 McTavish Street, Montreal, PQ H3A IHI CANADA

McKay, Jym M., Dept. of Speech Conmunication, University of Texas, Austin, TX 78712

McNeese State University, Serials Department, Frazar Memorial Library, Lake Charles, LA 70609

Menin, J. L., Language Laboratory, Rice University, Rayzor Hall 106, Houston, TX 77005

Messner, Jr., C. A., Modern Languages, Carleton College, Northfield, MN 55057

Metcalfe, John, Language Laboratory, Stanford University, Stanford, CA 94305

Miller, j. B., Wittenberg University, Springfield, OH 45501

Miller, William I., Modern Languages, University of Akron, 31001 in Hall, Akron, $\mathrm{OH} 44325$

Ministry-Culture \& Recreation, Citizenship Language C1asses, 454 University Avenue, Toronto, Ont M4G $1 Y 7$ CANADA 
Ministry-Culture \& Recreation, Resource Centre, 77 Bloor Street West, Toronto, Ont M7A 2R9 CANADA

Miura, Shogo, 72-121, Jike, Sayo-cho, Higashi-Hiroshima 724 JAPAN

Modern Language Association, Advertizing Manager, 62 Fifth Avenue, New York, NY 10011

MODERN LANGUAGE JOURNAL, Editor, Dept. of German, Ohio State University, 314 Cunz Hall, Columbus, $\mathrm{OH} 43210$

Modl in, Columbus M., Central Area Admin. Center, 6501 Lowl and Drive, Landover, MD 20786

Monash University, Periodicals, Clayton, Vic 3168 AUSTRALIA

Moorhead State University, Library-Serials Dept., Moorhead, MN 56560

Morrison, Robert R., Southern Missionary College, P.0. Box 475, Collegedale, TN 37315

Moseby, Erik, Tandberg A/S, P. O. Box 55 - Bogerud, Os 106 NORWAY

Moses, James 0., Austin State University, Box 13042, Nacogdoches, TX 75962

Nagoya Gakuin Daigaku, Gaikokugo Center, Kamishinano-Cho, Seto, Aichiken 480-12, MZ JAPAN

Nassau Community College, Library -Periodicals Unit, Stewart Avenue, Garden City, NY 11530

National Institute of Education, Serials Section, Educational Research Library, Washington, D.C. 20208

National Library of Australia, Prel iminary Processing, (NS 110/72), Canberra, ACT 2600 AUSTRALIA

National Library of Canada, Order Section, 395 Wellington Street, Ottawa, Ont KIA ON4 CANADA

National University of Singapore, Kent Ridge Library, Chief Librarian, Kent Ridge SINGAPORE

Nelson, R., College Department, John Wiley \& Sons, 605 Third Avenue, New York, NY 10016

Nicholson, C., Department of Education, 120 East 10th Street, Topeka, KS 66612

Nicholson, Cynthia, Language Laboratory, Boston College, Lyons 313, Chestnut Hill, MA 02167

Nielson, M., Education Department, 301 Centennial Mall South, Lincoln, NE 68509

Norrish, N., Language Laboratory, University of Victoria, Private Bag, Wellington NEW ZEALAND

North Texas State University, Acquisitions Services, P.0. Box 5188, Denton, TX 76203

Novak, Sigrid Scholtz, Modern Languages, McNeese State University, Lake Charles, LA 70609

Ochoa, A., Department of Education, $650 \mathrm{w}$ State St-Jordan B1dg, Boise, ID 83720

Odense Universitetsbib1, TSS. Campusvej SS, DK-5230 Odense M DENMARK

O'Donohue, Barbara, Hiroshima Bunkyo Womens College, Kabe Cho-Asa Kita Kucho, Hiroshima 731-92 JAPAN

0'Hearon, Sr. Marie Therese, Language Laboratory, College of White Plains, 78 North Broadway, White P1ains, NY 10603

Ohio State University, Library, Serials Division, 1858 Neil Avenue, Columbus, $O H$ 43210

OKlahoma Baptist Univ, Attn: Director, Language Laboratory, Shawnee, OK 74801

OKl ahoma State University, Edmon Low Library-Serials, Stiliwater, OK 74074

Ort, B., Department of Education, P. 0. Box 30008, Lansing, MI 48909

Oueilette, R. J., Language Lab., 207 I Bldg., SE Massachusetts Univ., 01d Westport Rd., N. Dartmouth, MA 02747

Pallister, Janis L., 211 State Street, Bowling Green, OH 43402

Parr, Susan, Language Laboratory, Juniata College, Huntingdon, PA 16652

Patron, Gloria A., Language Laboratory, University of New Orleans, Lake Front, New Orleans, LA 70122 
Pennsylvania State Library, Serial Records Section, Box 1601, Room 46, Harrisburg, PA 17126

Pensacola Junior College Library, 1000 College Boulevard, Pensacola, FL 32504

Penta, Richard M., Foreign Language Coordinator, Belmont School System, 38 Normandy Road, Lexington, MA 02173

Peterson, Gerald W., Dept. of Foreign Languages, University of Nevada, Reno, NV 89507

Peterson, Greg, Notre Dame Women's College, Shimogamo, Sakyo-ku, Kyoto 606 JAPAN

Petherbridge, D. L., University of Lethbridge, Lethbridge, Al ta T1K 3M4 CANADA

Pflanz, Barbara, Dept. of German, University of Redlands, Redl ands, CA 92373

Pierce, James A., Dept. of Engl ish, Del Mar College-East, Corpus Christi, TX 78404

Pineyro, R., Department of Education, 721 Capitol Mall, Sacramento, CA 95814

Pleasants, J. V., 2815 Treasure Is I and East, Memphis, TN 38115

Powell, Richard K., Teaching Materials-Learning Center, Andrews University, Berrien Springs, MI 49104

Prawer, Florence H., 10 Tobey Brook, Pittsford, NY 14534

Pritchard, C., SONY of Canada Ltd, 411 Gordon Baker Rd, Willowdale, ONT MEH 256 CANADA

Purdue University Libraries, PCF-Kardex 19573/Serials, West Lafayette, IN 47907

Queens College, Serials Librarian, Flushing, NY 11367

Ramsay, Patricia L., Language Laboratory, Eastern Michigan University, Ypsilanti, MI 48197

Rawlings, Ruth, 101 Kobuke Cho, Chiba Shi, Chiba Ken 281 JAPAN

Raymond, Walter, Atelier de langues, CEGEP Ste-Foy, 2410, Chemin Ste-Foy, Ste-Foy, Quebec GIV IT3 CANADA

Reeves, Mrs. Gaynor E., Language Laboratory, University of Newcastle 2308, Newcastle, NSW AUSTRALIA

Reimers, Theresia E., Department of Modern Languages, Hollins College, Hollins College, VA 24020

Renegar, Christopher, Language Laboratory, Occidental College, 1600 Campus Road, Los Angeles, CA 90041

Richardson, Martha R., Harvard University, Boylston Hall G-3, Cambridge, MA 02138

Richmond, Edmun B., Modern Language Dept., Georgia Institue of Technology, Atlanta, GA 30332

Riekehof, Lottie L., Dept. of Sign Communication, Gallaudet College, Kendall Green, Washington, D.C. 20002

Rijksunivers Te Groningen, Bibliotheek, Postbus 559, Oude Kijk In't Jatstraat 5, Groningen NETHERLANDS

Rivers, Wilga M., Department of Romance Languages, Harvard University, 207 Boyiston Hall, Cambridge, MA 02138

Rodewald, Janet $D$., Language Laboratory, Central Michigan University, Mt. Pleasant, MI 48858

Rollin, Marian B., 640 Wornwood Hill Rd., Storrs, CT 06268

Royalty, James E., Language Media Center, University of Maryland, College Park, MD 20742

Saga University, Honjyo-Machi, Saga City 840 MZ JAPAN

Sampon, Victor, Language Laboratory, Wayl and Academy, Beaver Dam, WI 53916

San Diego State University, Language Lab-42061001, San Diego, CA 92182

San Diego State University, Love Library-Serials, San Diego, CA 92182

Sananvanit, Ms. Kwanchai, SEA Enterprises Co., Ltd., Paris Theatre, 292/15-16 Luk Luang Road, Bangk ok 3 THAILAND

Sanchez-Berroa, Robert A., Language Laboratory, Wesleyan University, 116 Fisk Ha11, West Station, Middletown, CT 06457 
Sapporo Ika Daigaku, Toshokan S-1 W-17, Sapporo 060 (KIN) JAPAN

Sawyer, Isabel, Language, Linguistics \& Literature, Simon Fraser University, Burnaby BC CANADA

Saylor, Leslie G., Language Laboratory, University of Wisconsin, P.0. Box 413, Milwaukee, WI 53201

Schaedel, Gene E., Kay Elemetrics Corporation, 12 Maple Avenue, Pinebrook, NJ 07058

Schladweiler, K., Language Arts, Education Division, Kneip Building, Pierre, SD 57501

Schobel, Dianne, 7801 Sagamore Drive, Cincinnati, OH 45236

Schrader, Ernst, DEP Design Editing Prod., Inc., 15 East 26th St., Rm 1908A, New York, NY 10010

Schultz, David, Media Services, Lehman College-Corman B38, Bedford Park Boulevard, West, Bronx, NY 10468

Sclafani, Charles, Language Laboratory, Westchester Community College, Valhalla, NY 10595

SEAMEO Reg. Language Center, Librarian of Information Center, 30 Orange Grove Road, Singapore 1025 SINGAPORE

Seidel, J., Language Laboratory, University of Victoria, P. 0. Box 1700, Victoria BC V8H 2 Y2 CANADA

Seinan Gakuin University, Language Lab, 6-2-92 Nishijin Nishi-Ku, Fukuoka 814 JAPAN

Seng, Mark W., University of Texas, EDB 562 FLEC, Austin TX 78741

Sheehan, Joseph H., Language \& Culture Center-English, University of Houston, Houston, TX 77004

Simon Fraser University, Library-Acquisition Division, Burnaby BC V5A 1 S6 CANADA

Slagle, Maryanne, Learning Center, Buena Vista College, Storm Lake, IA 50588

Smith, G., Foreign Language Specialist, Education Language Department, Memphis State University, Memphis, TN 38152

Smith, Philip D., Languages, West Chester State College, West Chester, PA 19380

Smith, William Fi int, Dept. of Modern Languages, Purdue University, LaFayette, IN 47907

Smither, William J., Dept. of Spanish \& Portuguese, Tulane University, New Orleans, LA 70118

Sommer, Ursula, Friendship Library, Fairleigh Dickinson University, 285 Madison Avenue, Madison, NJ 07940

Soumokil, P., Bilingual Education, Hathaway Building, Cheyene, Wr 82002

Southern Illinois University, Morris Library-Periodicals, Carbondale, IL 62901

Spinks, 0. R., Humanities B-16, SUNY-Albany, Albany, NY 12222

St. Michael's College, Attn: Norman Lacharite, Director, International Studies, Winooski, VT 05404

Stadt \& Universitatsbibl, Bockenheimer Landstr 134, 6000 Frankfurt Am Main WEST GERMANY

Staley, H., Education Department, P. 0. Box 771, Jackson MS 39205

Stanford University, Library-Serials, Stanford, CA 94305

State University College, Butler Library, Box P, 1300 Elmwood Avenue, Buffalo, NY 14222

Steffensen, Kjell, Tandberg of America, Inc., Labriola Court, Armonk, NY 10504

Strauber, Irv, Telex Communications Inc., P.0. Box 182, 5339 Newcastie \#202, Encino, CA 91316

SUNY-Binghamton, Library -Serial Section, Vestal Parkway East, Binghamton, NY 13901

Swanson, Severin A., Language Laboratory, University of Wisconsin, Oshkosh, WI 54091

Tanner, Jackie, Language Learning Technology/SLL, Georgetown University, Washington, DC 20057

Taylor, Jr., Walter C., Department of Spanish, Upsala College, East Orange, NJ 07019 
Teachers College Library, Serials Dept., Box 307, 525 West 120th Street, New York, NY 10027

Texas Tech University, Germanic \& Slavic Languages, P.0. Box 4579, Lubbock, TX 79409

Thibault, Claude, Notre-Dame-de-Foy, Cap Rouge, PQ GOA IKO CANADA

Thomas, J. E., Learning Laboratory, Virginia Polytechnic Institute, Blacksburg, VA 24061

Tokyo-Seitoku-Tanki-Daigaku, Library (MZ), 7-13, Jujodai-1-Chome, Kita-Ku, Tokyo JAPAN

Tracy, Robert K., Language Laboratory, Ridgewood High School, Ridgewood, NJ 07450

Trometer, Ruth, Language Lab, Mass. Inst. Technology, Bl dg No. 14N-0641, Cambridge, MA 02139

Unme1, Carolyn M., Valley View High School, 6027 Farmersville Pike, Germantown, $\mathrm{OH} 45327$

Union College, Library, $3800 \mathrm{~S}$. 48th Street, Lincoln, NE 68506

Universidad de Concepcion, Biblioteca Central, Casilia 1807, Concepcion CHILE

Universitat Bern, Verwal tung, Hochschul strasse 4, 3012 Bern SWITZERLAND

Universitatsbibliothek, Zeitschriften-FGSZ, Universitatsstrasse 4, $8520 \mathrm{Erl}$ angen WEST GERMANY

Universite de Neuchate1, Chaire de Linguistique Generale, Neuchatel, 2000 SHITZERLAND

Universite de Strasbourg, Inst Linguistque Appliquee, Laboratoires de Langues, 22, rue Descartes, Strasbourg-Cedex 67084 FRANCE

Universiteitsbibliotheek 1439, A. 324. 639, Eras Muslaan 36, Nijmegen 1 NETHERLANDS

University Microfilms International, Serials Development, Manager, Publisher Relations \& Development, 300 North Zeeb Road, Ann Arbor, MI 48106

University of Alberta, Attn: C. Dechaine, Language Lab, Edmonton, Alta T6G 2E1 CANADA

University of California, Language Laboratory, Attn: Director, B-40 Dwinelle Hall, Berkeley, CA 94720

University of California-San Diego, Language Laboratory C-008, LaJolla, CA 92093

University of Colorado, Library-Serials, Boulder, CO 80309

University of Dayton, Periodical Dept., Roesch Library, 300 College Park Avenue, Dayton, $\mathrm{OH} 45469$

University of Georgia, Periodicals Desk-LT, Libraries, Athens, GA 30602

University of Illinois, Language Learning Laboratory, G-70 FLB, Urbana, IL 61801

University of Illinois-Urbana, Serials Dept., Library-Fax, Urbana, IL 61801

University of Kansas Libraries, Periodicals-Serials, Lawrence, KS 66045

University of Kentucky, Language Laboratory, Cl assroom Building 312, Lexington, KY 40506

University of Louisville, Attn: Mrs. Spradlin, Serials Department-Library, Louisville, KY 40292

University of Melbourne, Honwood Language Centre, Medlay Building, West Tower, Parkville, Vic 3052 AUSTRALIA

University of Minnesota, Libraries-Serial Records, Minneapolis, MN 55455

University of Missouri, Library-Serials, Columbia, MO 65201

University of Nebraska, Eppley Library-Periodicals, 60th \& Dodge Sts., Omaha, NE 68182

University of Nevada, Library-Periodicals, Las Vegas, NV 89154

University of Nevada, Library-Serials, Reno, NV 89557

University of Newcastle, Serials, Auchmuty Library, Newcastle, NSW 2308 AUSTRALIA 
University of Queensland, Serials Librarian, Main Library, St. Lucia, Qnslnd. 4067 AUSTRALIA

University of South Africa-Port Elizabeth, Library-Periodicals Dept., Private Bag 6058, Port Elizabeth 6000 SOUTH AFRICA

University of South Dakota, ID Weeks Library, Vermillion, SD 57069

University of SW Louisiana, Dupre Library-Serials, 302 East St. Mary Blvd., Lafayette, LA 70504

University of St. Andrews, Attn: Ms. Konn, Modern Language Library, Buchannon Bldg - Union Street, St. Andrews, Fife KY16 9PH SCOTLAND

University of Stellenbosch, Library, Private Bag 5036, Stellenbosch 7600 SOUTH AFRICA

University of Sydney, Serials Division 134881, Fischer Library, Sydney, NSW 2006 AUSTRALIA

University of Toledo, Carlson Library-Serials, Toledo, $\mathrm{OH} 43606$

University of Turku, Language Centre, 20500 Turku 50 FINLAND

University of Western Ontario, Periodicals-General Library, London, Ont N6A 3K7 CANADA

University of Wisconsin, Library-Serials, 728 State Street, Madison, WI 53706

University of Wisconsin-Milwaukee, Library, Serials Department, P.0. Box 604, Milwaukee, WI 53201

University of Wisconsin-Parkside, Library-Serials, Wood Road, Kenosha, WI 53141

University of Zimbabwe, Language Laboratory \#3643, P. 0. Box MP 167, Mount Pleasant, Salisbury ZIMBABWE

USAF Academy, Library DFSLBS, Colorado Springs, CO 80840

Van 0lphen, H., Language Laboratory, Batts Hall 1, Austin, TX 78712

Vernick, Judy A., Language Laboratory, University of Pittsburgh, $732 \mathrm{C}$ of L, Pittsburgh, PA 15260

Vijayakumar, E. K., Thurber Comprehensive High School, 15 Orilla Park, Red Deer, A1 ta T4N 5 A6 CANADA

Virginia Polytechnic Institute, Newman Library-Serials, Bl acksburg, VA 24061

Voge, Wilfried, Language Laboratory, University of California, Humanities Hall 263, Irvine, CA 92717

Waguespack, P., Language Education, P. O. Box 44064, Baton Rouge, LA 70804

Walsh, Terence M., St. Paul's School, Pleasant St., Concord, NH 03301

Warkentin, Henry, Foreign Language Department, Shippensburg State College, Shippensburg, PA 17257

Warriner, H., Billingual Education, P. 0. Box 6Q, Richmond, VA 23216

Watson, John A., Language Laboratory, Virginia Union University, Richmond, VA 23220

Wayne County Intermed Sch Dist, Attn: Ms. B. Katz, Info. Svc.-Prof. Res. Ctr., 33400 Van Born Rd., Wayne, MI 48184

Weible, David M., Audio Information Service, Univ. Illinois-Chicago Circle, Box 4348, Chicago, IL 60680

Weinkauf, Arnold L., Dept. of Languages, Michigan Technological University, Houghton, MI 49931

WERA Inc., Attn: Jill Nickson, 5761 Buckingham Pkwy, Culver City, CA 90230

WERA Inc., Attn: Chris Wheaton, 5761 Buckingham Pkwy., Culver City, CA 90230

Wessel, Jan, Tandberg of America, Inc., Labriola Court, Armonk, NY 10504

West Chester State College, Francis $H$. Green Library, Department of Education, West Chester, PA 19380

West Texas State University, Cornette Library, Box 748, W.T. Station, Canyon, TX 79016

Wickert, Gabriele, Language Resource Center, Manhattanville College, Purchase, NY 10577

Wiese, Peter, Language Laboratory, Southern Connecticut College, 501 Crescent Street, New Haven, CT 06515

Wilde, Karen, 1605 N. Fillmore St., Arlington, VA 22201 
Willecke, F. H., Foreign Language Department, Wagner College, Staten Is Iand, NY 10301

Williams, Charles B., Language Laboratory, State University of New York, Oswego, NY 13126

Willis, Robert M., Communications - Arts \& Sciences, 829 Shink in Hall, 50 West 4 th Street, New York, NY 10003

Williston, Jacquel ine, Language Laboratory, Connecticut College, P. 0. Box 1524, New London, CT 06320

Wilton High School, Library, 395 Danbury Road, Wilton, CT 06897

Wipf, Joseph, 121 East. Knox Drive, West Lafayette, IN 47906

Wyatt, David H., American Language Academy, Catholic University, Washington, D.C. 20064

Wynn, Walter W., 975 Ventura Avenue, Albany, CA 94707

Yamashita, Kikuko, 23 Hancock Street, Somerville, MA 02144

York University Libraries, Central Serials Records, 4700 Keele Street, Downsview, Ont M3J 2R2 CANADA

Zaetta, R., Department of Instruction, Townsend Building, Dover, DE 19901

Zimnerman, Mrs. M., Language Laboratory, Amherst College, Amherst, MA 01002

Zimmerman, Sr. Dorothy M., Fourier Laboratory, Mount Mary College, Milwaukee, WI 53222

\section{GEOGRAPHICAL LIST}

ALABAMA

Cox, Shirley 0., Language Laboratory, Auburn University, Auburn, AL 36830

Hirsch, Bernice G., Foreign Language Laboratory, Samford University, 800 Lakeshore Drive, Birmingham, AL 35229

Howard, J., Department of Education, 111 Coliseum Boulevard, Montgomery, AL 36109

ARIZONA

Arizona State University, Library-Periodicals, Tempe, AZ 85281

Maynes, J., Department of Education, 1535 West Jefferson, Phoenix, AZ 85007

ARKANSAS

Cheatham, Rosalie M., Language Laboratory, University of Arkansas-Little Rock, 6405 Evergreen Rd., Little Rock, AR 72207

Hudson, G., Department of Education, Little Rock, AR 72201

CALIFORNIA

Barr, Charles J., Language Laboratory, St. John's Seminary College, 5118 East Seminary Road, Camarillo, CA 93010

Bauman, Ronald A., Modern European Languages, Pomoma College, Claremont, CA 91711

Berry, Kenneth Russell, Media Services, Santa Barbara City College, 721 Cliff Drive, Santa Barbara, CA 93109

Bridgers, W. K., Learning Laboratories, University of California, Phelps Hall, Santa Barbara, CA 93106

Brinton, Donna, English IESL, UCLA, $405 \mathrm{Hilgard}$ Ave., Los Angeles, CA 90024

Calif Polytech State Univ., Library-Periodicals, San Luis Obispo, CA 93407

Comet, Honore, Language Lab-Suite 500, All-World Language Institute, 280 South Beverly Drive, Beverly Hills, CA 90212

DeMers, Irene, Foreign Languages, Harvard School, 3700 Coldwater Canyon Road, North Hollywood, CA 91604

DLIWC ACademic Library, DLIFLC-Building 618, Presidio, M/F DAKF 03-80-M-3315, Monterey, CA 93940 
Educational Electronics-California, Attn: Bernard Keach, 213 North Cedar Avenue, Inglewood, CA 90301

Ford, Kathleen, Instructional Laboratory, UCLA, 362 Royce Hall, Los Angeles, CA 90024

Forth, Joli, 973 Kingston Avenue, Piedmont, CA 94611

Froehilich, Jurgen, Language Laboratory, Scripps College, Cl aremont, CA 91711

Ha11, Bunelle M., Monterey Inst. Int'1. Studies, 425 Van Buren Street, Monterey, CA 93940

Hilts, Margarete, La Sierra College, Loma Linda University, Riverside, CA 92515

Kessler, Michael, Lang Lab-Humanities, San Francisco State Univ, 1600 Holloway, San FrancisCo, CA 94132

Kirk, David, 124 Chace Street, Santa Cruz, CA 95060

LANGUAGE \& LANGUAGE BEHAVIOR ABSTRACTS, P.0. Box 22206, San Diego, CA 92122

Leonhardt-Hendriks, Helga M., Foreign Language Instructor, 640 San Elijo Street, San Diego, CA 92106

Long Beach City College, Attn: Lorraine Yocky, Foreign Language Dept., 4901 East Carson Street, Long Beach, CA 90808

Metcalfe, John, Language Laboratory, Stanford University, Stanford, CA 94305

Pflanz, Barbara, Dept. of German, University of Redlands, Redlands, CA 92373

Pineyro, R., Department of Education, 721 Capitol Mall, Sacramento, CA 95814

Renegar, Christopher, Language Laboratory, Occidental College, 1600 Campus Road, Los Angeles, CA 90041

San Diego State University, Language Lab-42061001, San Diego, CA 92182

San Diego State University, Love Library-Serials, San Diego, CA 92182

Stanford University, Library-Serials, Stanford, CA 94305

Strauber, Irv, Telex Commitcations Inc., P.0. Box 182, 5339 Newcastle \#202, Encino, $\mathrm{CA} 91316$

University of California, Language Laboratory, Attn: Acting Director, B-40 Dwinelle Hall, Berkeley, CA 94720

University of Cailifornia-San Diego, Language Laboratory C-008, LaJolla, CA 92093

Voge, Wilfried, Language Laboratory, University of California, Humanities Hall 263, Irvine, CA 92717

WERA Inc., Attn: Jill Nickson, 5761 Buckingham Pkwy, Culver City, CA 90230

WERA Inc., Attn: Chris Wheaton, 5761 Buckingham Pkwy., Culver City, CA 90230

Wynn, Walter W., 975 Ventura Avenue, Albany, CA 94707

\section{COLORADO}

Butler, Samuel R., 1095 South Downing Street, Denver, CO 80209

Gilmore, Roger H., Foreign Languages, Colorado State University, Fort Collins, CO 80523

Macinko, John, Language Laboratories, University of Colorado, 302 old Main Building, Boulder, CO 80309

USAF Academy, Library DFSLBS, Colorado Springs, CO 80840

University of Colorado, Library-Serials, Boulder, CO 80309

\section{CONNECTICUT}

Capretz, Pierre J., Language Laboratory, Yale University, 111 Grove Street, New Haven, CT 06510

Language Instruction Supervisor, Bilingual Education, P. 0. Box 2219, Hartford, CT 06115

Rollin, Marian B., 640 Wormwood Hill Rd., Storrs, CT 06268

Sanchez-Berroa, Robert A., Language Laboratory, Wesleyan University, 116 Fisk Hall, West Station, Middletown, CT 06457

Wiese, Peter, Language Laboratory, Southern Connecticut College, 501 Crescent Street, New Haven, CT 06515

Williston, Jacquel ine, Language Laboratory, Connecticut College, P. 0. Box 1524, New London, CT 06320

Wilton High School, Library, 395 Danbury Road, Wilton, CT 06897 


\section{DELAWARE}

Zaetta, R., Department of Instruction, Townsend Building, Dover, DE 19901

DISTRICT OF COLUMBIA

Assoc Ed Comm \& Tech, Attn: Charles Van Horn, 1126 Sixteenth St., N.W., Washington, D.C. 20036

Caldwell, Wm. A., Language Laboratory, The American University, Massachusetts \& Nebraska Aves. N.W., Washington, D.C. 20016

ERIC Exchange, LR, Center for Applied Linguistics, 3520 Prospect Street, NW, Washington, D.C. 20007

Howard University, Language Lab/903301, Room 361 Locke Hal1, 2441 6th Street, N.W., Washington, O.C. 20059

Howard University Library, Acquisitions Dept., Serials, Room \#105, Washington, D.C. 20059

Laroche, J., Languages, D. C. Public Schools, 415 12th Street, N.W., Washington, D.C. 20004

Library of Congress, Order Division, Continuations Unit, Washington, D.C. 20540

Mazzeo, Guido E., Romance Languages \& Literatures, George Washington University, 2115 G Street, N.W., Rm. 411, Washington, D.C. 20052

National Institute of Education, Serials Section, Educational Research Library, Washington, D.C. 20208

Riekehof, Lottie L., Dept. of Sign Communication, Gallaudet College, Kendall Green, Washington, D.C. 20002

Tanner, Jackie, Language Learning Technology/SLL, Georgetown University, Washington, DC 20057

Wyatt, David H., American Language Acadeny, Catholic University, Washington, D.C. 20064

\section{FLORIDA}

Arnold, Robert L., Instructional Resources, University of Central Florida, Box 25,000 , Orlando, FL 32816

Cate, Bob, P.0. Box 260133, Tampa, FL 33685

Clifford, Billy B., 5353 Arl ington Expressway, Apt. 9N, Jacksonville, FL 32211

Florida State University, Serials, Strozier Library, Tallahassee, FL 32306

Frechette, Ernest A., Foreign Language Education, Fiorida State University, Tallahassee, FL 32306

Goldsworthy, Thomas, P.0. Box 537, Odessa, FL 33556

LHTEC, 905 Lindsey Hopk ins Library, 1410 N.E. 2nd Avenue, Mi ani, FL 33132

Pensacola Junior Coll Lib, 1000 College Boulevard, Pensacola, FL 32504

\section{GEORGIA}

Ellison, Keith, Department of Foreign Languages, West Georgia College, Caroliton, GA 30118

Feagin, C., Department of Education, Education Annex-Trinity, Atlanta, GA 30303

Herrera, Lazaro M., Language Laboratory, Westninster Schools, 1424 West Paces Ferry, N.W., At I anta, GA 30327

Richmond, Edmun B., Modern Language Dept., Georgia Institue of Technology, Atlanta, GA 30332

University of Georgia, Periodicals Desk-LT, Libraries, Athens, GA 30602

GUAM

Barnes, J., Department of Education, Agana GUAM 96910

\section{HAWAII}

Cassidy, Maureen, Foreign Languages, Dept. of Education, 1270 Queen Emma St, Rm. 1201, Honolulu, HI 96813

Chang, Gerald K. J., Foreign Language Laboratory, University of Hawaii, 1890 East-Kest Road, Honolulu, HI 96822

Genmer, Bi11, Kamehameha Schools, Keona-Lower Campus, Honolulu, HI 96817 
Harvey, Edward, Hawaif Campus, Brigham Young University, Box 33 BYU-HC, Laie, HI 96762

Kodama, Paul, Learning Assistance Center, Hawaif Pacific College, 1164 Bishop Street, Honolulu, HI 96813

IDAHO

Hughett, Harvey, Foreign Languages \& Literatures, University of Idaho, Moscow, ID 83843

Ochoa, A., Department of Education, $650 \mathrm{~W}$ State St-Jordan B1dg, Boise, ID 83720

ILLINOIS

Bailey, Leslie F., Language Laboratorles-48B Kresge, Northwestern University, 1859 Sheridan Rd., Evanston, IL 60201

Gillespie, Netta, Language Laboratory, University of Illinois, G-89 Foreign Languages, Urbana, IL 61801

Governors State University, Library/Serials-Kardex, Park Forest South, IL 60466

Griffith, P., Education, 100 North First Street, Springfield, IL 62777

Heather, Anthony J., Language Resource Center, Loyola University, Chicago, IL 60626

Heilenman, Laura K., Dept. of French \& Italian, Northwestern University, Evanston, IL 60201

HISPANIA, Attn: Albert Turner, Glenbrook South High School, 4000 West Lake Avenue, Glenview, IL 60025

Southern Illinois University, Morris Library-Periodicals, Carbondale, IL 62901

University of Illinois, Language Learning Laboratory, G-70 FLB, Urbana, IL 61801

University of Illinois-Urbana, Serials Dept., Library-Fax, Urbana, IL 61801

Weible, David M., Audio Information Service, Univ. Illinois-Chicago Circle, Box 4348, Chicago, IL 60680

INDIANA

Ball State University, Dept. of Library Service, Muncie, IN 47306

Bartz, W., Public Instruction, State House 229, Indianapolis, IN 46204

Cochran, Clay, 205 North Walnut Street, Brazil, IN 47934

McCune, Jerry, Language Laboratory, Indiana University, Ballantine Hall 120, Blocmington, IN 47401

Purdue University Libraries, PCF-Kardex 19573/Serials, West Lafayette, IN 47907

Smith, William Flint, Dept. of Modern Languages, Purdue University, LaFayette, IN 47907

Wipf, Joseph, 121 East Knox Orive, West Lafayette, IN 47906

IOWA

Irving, Evelyn Uhrhan, 2508 Glen Elm Drive, N.E., Cedar Rapids, IA 52402

Slagle, Maryanne, Learning Center, Buena Vista College, Storm Lake, IA 50588

\section{KANSAS}

Garinger, Ermal E., Language Laboratories, University of Kansas, 4069 Wescoe Hall, Lawrence, KS 66045

Nicholson, C., Department of Education, 120 East 10th Street, Topeka, KS 66612

University of Kansas Libraries, Periodicals-Serials, Lawrence, KS 66045

\section{KENTUCKY}

Altman, Howard B., Dept. of Modern Languages, University of Louisville, Louisville, KY 40292

Brown, C. P., Foreign Language Department, Western Kentucky University, Ivan Wilson Building 251, Bowling Green, KY 42101

Gwinn, Joel A., Physics Department, University of Louisville, Louisville, KY 40292

Lally, Kenneth, 3222 Murray Hill Pike, Louisville, KY 40222 
University of Kentucky, Language Laboratory, Classroom Building 312, Lexington, KY 40506

University of Louisville, Attn: Mrs. Spradlin, Serials Department-Library, Louisville, KY 40292

\section{LOUISIANA}

Abed, A. Z., Foreign Language Laboratory, Louisiana State University, Baton Rouge, LA 70803

McNeese State University, Serials Department, Frazar Memorial Library, Lake Charles, LA 70609

Novak, Sigrid Scholtz, Modern Languages, McNeese State University, Lake Charles, LA 70609

Patron, Gloria A., Language Laboratory, University of New Orleans, Lake Front, New Orleans, LA 70122

Smither, William J., Dept. of Spanish \& Portuguese, Tulane University, New Orleans, LA 70118

University of SW Louisiana, Dupre Library-Serials, 302 East St. Mary Blvd., LaFayette, LA 70504

Waguespack, P., Language Education, P. 0. Box 44064, Baton Rouge, LA 70804

MARYLAND

Aulestia, Victor H., Language Media Center, University of Maryland, 5401 Wilkins Avenue, Baltimore, MD 21228

Beusch, A., Department of Education, P. 0. Box 8717, BWI Airport, Baltimore, MD 21240

Bowie State College, Pullen Library-Periodicals, Bowie, MD 20715

Clark, D. J., Language Lab, Gilman Hall, Johns Hopk ins University, Baltimore, MD 21218

DuVerlie, Claude, Modern Languages \& Linguistics, UMBC, Catonsville, MD 21228

Ford, Penelope, Instructional Media Resources, Language Ctr./Univ. of Maryland, Catonsville, MD 21228

Kennedy, Dora F., Foreign Language Department, Prince Georges' Public School, 4806 Harvard Road, College Park, MD 20740

Magi11, Robert, Language Lab, Modern Languages, Towson State University, Baltimore, MD 21204

Modlin, Columbus M., Central Area Admin. Center, 6501 Lowl and Drive, Landover, MD 20786

Royalty, James E., Language Media Center, University of Maryland, College Park, MD 20742

\section{MASSACHUSETTS}

Boudreau, Jacqueline, Language Lab., Wheaton College, Norton, MA 02766

Carlson, Florence, AV Director, Wellesley College, Wellesley, MA 02181

Department of Education, Attn: Director, Bilingual Education, Boston, MA 02111

Earle, Betty B., 59 Henderson Street, Needham, MA 02192

Enos, Jacqueline, Foreign Language Dept., Bridgewater State College, Bridgewater, MA 02324

Fritsch, Hans J., P. 0. Box 61, Whately, MA 01093

Gore, Peter B., P. 0. Box 11, Deerfield, MA 01342

Heinle \& Heinle Publishers, Inc., Attn: Charles Heinle, 51 sleeper Street, Boston, MA 02210

Nicholson, Cynthia, Language Laboratory, Boston College, Lyons 313, Chestnut Hill, MA 02167

Ouellette, R. J., Language Lab., 207 I Bldg., SE Massachusetts Univ., 01d Westport Rd., N. Dartmouth, MA 02747

Penta, Richard $M$., Foreign Language Coordinator, Belmont School System, 38 Normandy Road, Lexington, MA 02173

Richardson, Martha R., Harvard University, Boylston Hall G-3, Cambridge, MA 02138 
Rivers, Wilga M., Department of Romance Languages, Harvard University, 207 Boylston Hall, Cambridge, MA 02138

Trometer, Ruth, Language Lab, Mass. Inst. Technology, Bldg No. 14N-0641, Cambridge, MA 02139

Yamashita, Kikuko, 23 Hancock Street, Somerville, MA 02144

Zimmerman, Mrs. M., Language Laboratory, Amherst College, Amherst, MA 01002

MICHIGAN

Beaudoin, John R., Language Laboratory, Western Michigan University, Kalamazoo, MI 49008

Calvin College Library, 3207 Burton, S.E., Grand Rapids, MI 49506

Mason, Richard G., Humanities Dept., Michigan Technological University, Houghton, MI 49931

Ort, B., Department of Education, P. O. Box 30008, Lansing, MI 48909

Powell, Richard K., Teaching Materials-Learning Center, Andrews University, Berrien Springs, MI 49104

Ramsay, Patricla L., Language Laboratory, Eastern Michigan University, Ypsilanti, MI 48197

Rodewald, Janet D., Language Laboratory, Central Michigan University, Mt. Pleasant, MI 48858

University Microfilms International, Serials Development, Manager, Publisher Relations \& Development, 300 North Zeeb Road, Ann Arbor, MI 48106

Wayne County Intermed Sch Dist, Attn: Ms. B. Katz, Info. Svc.-Prof. Res. Ctr., 33400 Van Born Rd., Wayne, MI 48184

Weinkauf, Arnold L., Dept. of Languages, Michigan Technological University, Houghton, MI 49931

MINNESOTA

Allen, Wendy W., Language Laboratory, Saint 01 af College, Northfield, MN 55057

Carleton College, Library, Northfield, MN 55057

Jebe, S., Department of Education, 649 Capitol Square, 550 Cedar Street, St. $P$ aul, MN 55101

Johnson, Tom, Audio-Visual Marketing, TELEX COMMUNICATIONS, INC., 9600 Aldrich Avenue South, Minneapol is, MN 55420

Lange, Dale L., 130B Peik Hail, University of Minnesota, Minneapolis, MN 55455

Messner, Jr., C. A., Modern Languages, Carleton College, Northfield, MN 55057

Moorhead State University, Library-Serials Dept., Moorhead, MN 56560

University of Minnesota, Libraries-Serial Records, Minneapolis, MN 55455

MISSISSIPPI

Staley, H., Education Department, P. O. Box 771, Jackson MS 39205

MISSOURI

Berndt, Robert J, Dept of Foreign Language \#4140, SW Missouri State University, Springfield, MO 65802

de Johnson, Teresa, Dept. of Modern Languages, St. Louis University, St. Louis, MO 63103

Francis, Larry P., Intensive English Program, University of Missouri, 228 Gentry, Columbia, Mo 65201

King, R., Education Department, P. O. Box 480, Jefferson City, Mo 65102

Kump, Richard J., 1822 Ricardo Drive, Cape Girardeau, M0 63701

University of Missouri, Library-Serials, Columbia, MO 65201

MONTANA

Eastern Montana College, Library-Serials Dept., Billings, MT 59101

Jackson, D., Second Languages, Public Instruction, Helena MT 59601

NEBRASKA

Coffey, Thomas F., Classics \& Modern Languages, Creighton University, Omaha, NE 68178 
Conner, Maurice W., Central States Conference, University of Nebraska, P.0. Box 688 , Omaha, NE 68101

Dalstrom, E. Kay, Foreign Languages, University of Nebraska, Omaha, NE 68112

Gilde, Hans M., University of Nebraska, Oldfather 1111, Lincoln, NE 68588

Nielson, M., Education Department, 301 Centennial Mall South, Lincoln, NE 68509

Union College, Library, 3800 S. 48th Street, Lincoln, NE 68506

University of Nebraska, Eppley Library-Periodicals, 60th \& Dodge Sts., Omaha, NE 68182

NEVADA

Abrams, W., Education Department, Capital Complex, Carson City, NV 89710

Peterson, Gerald W., Dept. of Foreign Languages, University of Nevada, Reno, NV 89507

University of Nevada, Library-Periodicals, Las Vegas, NV 89154

University of Nevada, Library-Serials, Reno, NV 89557

\section{NEW HAMPSHIRE}

Bowen, Louise H., 201 Bartlett Ha11, Dartmouth College, Hanover, NH 03755

Fournier, R., Bilingual Education, 64 North Main Street, Third Floor, Concord, NH 03301

Goulet, Sr. Noella, Notre Dame College, 2321 Elm Street, Manchester, NH 03104

Walsh, Terence M., St. Paul's School, Pleasant St., Concord, NH 03301

NEW JERSEY

Alyta, Kenneth J., International Programs Office, Stevens Institute of Technology, Hoboken, NJ 07030

Frost, Arthur' $F$., Language Laboratory, Princeton University, 304 E. Pyne Building, Princeton, NJ 08540

Schaedel, Gene E., Kay Elemetrics Corporation, 12 Maple Avenue, Pinebrook, NJ 07058

Sommer, Ursula, Friendship Library, Fairleigh Dickinson University, 285 Madison Avenue, Madison, NJ 07940

Taylor, Jr., Walter C., Department of Spanish, Upsala College, East Orange, NJ 07019

Tracy, Robert K., Language Laboratory, Ridgewood High School, Ridgewood, NJ 07450

NEW YORK

ADFL BULLETIN, Editor, 62 Fifth Avenue, New York, NY 10011

Bator, Joseph W., Language Development, Pan American World Airways, Kennedy International Airport, Jamaica, NY 11430

Bronx Community College, Library-Learning Center, West 181st Street, Bronx; NY 10453

Criminale, Leonard R., Language Laboratory, Elmira College, Elmira, NY 14901

Dammer, $P$., Foreign Language Education, Education Department, Albany, NY 12234

de la Portilla, Marta, Spanish Department, Manhattanville College, Purchase Street, Purchase, NY 10577

Goldsmith, Stanley, Language Laboratory, Columbia University, 101-B Philosophy Hall, New York, NY 10027

Kruse, Jr., E. L., 733 Kirkwood Drive, Grand Island, NY 14072

Levinson, Bernice, Dept. of Modern Languages, Brookiyn College-CUNY, Brooklyn, NY 11210

Mandelson, Leonore R., 10 Tobey Brook, Pittsford, NY 14534

Marino, Anna, Language Laboratory, Hunter College, 695 Park Avenue, Box 375, New York, NY 10021

McCarty, Joan, Language Laboratory, 106 Calkins, Hofstra University, 106 Calkins Ha11, Hempstead, NY 11550

Modern Language Association, Advertizing Manager, 62 Fifth Avenue, New York, NY 10011 
Nassau Community College, Library-Periodicals Unit, Stewart Avenue, Garden City, NY 11530

Nelson, R., College Department, John Wiley \& Sons, 605 Third Avenue, New York, NY 10016

$0^{\prime}$ Hearon, Sr. Marie Therese, Language Laboratory, College of White Plains, 78 North Broadway, White Plains, NY 10603

Prawer, Florence H., 10 Tobey Brook, Pittsford, NY 14534

Queens College, Serials Librarian, Flushing, NY 11367 NY 13901

Schrader, Ernst, DEP Design Editing Prod., Inc., 15 East 26th St., Rm 1908A, New York, NY 10010

Schultz, David, Media Services, Lehman College-Corman B38, Bedford Park Boulevard, West, Bronx, NY 10468

Sclafani, Charles, Language Laboratory, Westchester Community College, Valhalla, NY 10595

Spinks, 0. R., Humanities B-16, SUNY-Albany, Albany, NY 12222

State University College, Butler Library, Box P, 1300 Elmwood Avenue, Buffalo, NY 14222

Steffensen, Kjell, Tandberg of America, Inc., Labriola Court, Armonk, NY 10504

SUNY-Binghamton, Library-Serial Section, Vestal Parkway East, Binghamton,

Teachers College Library, Serials Dept., Box 307, 525 West 120th Street, New York, NY 10027

Wessel, Jan, Tandberg of America, Inc., Labriola Court, Armonk, NY 10504

Wickert, Gabriele, Language Resource Center, Manhattanville College, Purchase, NY 10577

Willecke, F. H., Foreign Language Department, Wagner College, Staten Island, NY 10301

Williams, Charles B., Language Laboratory, State University of New York, Oswego, NY 13126

Willis, Robert M., Communications -Arts \& Sciences, 829 Shimk in Hall, 50 West 4 th Street, New York, NY 10003

NORTH CAROLINA

Appalachian State University, Serials Department, Belk Library, Boone, NC 28608

D. H. Hill Library, Periodicals Service Center, Box 5007-NC State, Raleigh, NC 27650

Infante, J., Languages, Public Instruction Department, Raleigh, NC 27611

LoRe, A. G., Romance Languages, University of North Carol ina, 323 Dey Hall, Chapel Hill, NC 27614

NORTH DAKOTA

Lacher, S., Languages, Public Instruction, Capitol Building, Bi smarck, ND 58505

OHIO

Baker, R., Foreign Language Supervisor, Education Department, 65 South Front Street, Columbus, OH 43215

Boyden, Patrick C., Self-Instruction Center, Kent State University, 265 Library, Kent, $\mathrm{OH} 44242$

Cleveland State University, Library-Serials, 1860 E. 22nd Street, Cleveland, OH 44115

Coltharp, Jerry, A/V Service, 340 Gaskill Hall, Miami University, Oxford, OH 45056

Cormier, Ramona, 149 Baldwin, Bowling Green, $\mathrm{OH} 43402$

Frank, Stephania E., University Hall, Bowl ing Green State University, Bowl ing Green, $\mathrm{OH} 43402$

Hartwe11, Jerry, Instructional Media Center, Bowling Green State University, Bowling Green, $\mathrm{OH} 43403$

HISPANIA, Editor, Romance Languages, University of Cincinnati, Cincinnati, $\mathrm{OH}$ 45221 
Jackson, Gordon, 257 Cliffriew Drive, Gahanna, OH 43230

Loud, Mary, Foreign Languages, Youngstown State University, Youngstown, $\mathrm{OH}$ 44555

Marietta College, Modern Language Department, Marietta, OH 45760

Miller, J. B., Wittenberg University, Springfield, $\mathrm{OH} 45501$

Miller, William I., Modern Languages, University of Akron, 31001 in Hall, Akron, OH 44325

MODERN LANGUAGE JOURNAL, Editor, Dept. of German, Ohio State University, 314 Cunz Hall, Columbus, OH 43210

Ohio State University, Library, Serials Division, 1858 Neil Avenue, Columbus, OH 43210

Pallister, Janis L., 211 State Street, Bowling Green, $\mathrm{OH} 43402$

Schobel, Dianne, 7801 Sagamore Drive, Cincinnati, $\mathrm{OH} 45236$

Ummel, Carolyn M., Valley View High School, 6027 Farmersville Pike, Germantown, $\mathrm{OH} 45327$

University of Dayton, Periodical Dept., Roesch Library, 300 College Park Avenue, Dayton, $\mathrm{OH} 45469$

University of Toledo, Carlson Library-Serials, Toledo, OH 43606

OKLAKOMA

Educational Media Division, Attn: Jim Goodin, P.0. Box 20604, OKlahoma City, OK 73156

Educational Media Division, Attn: Shelby Hardage, P.0. Box 20604, Oklahoma City, OK 73156

Educational Media Division, Attn: R. Pohlmeier, P.0. Box 20604, Oklahoma City, OK 73156

Oklahoma Baptist Univ, Attn: Director, Language Laboratory, Shawnee, OK 74801

OKlahoma State University, Edmon Low Library-Serials, Stillwater, OK 74074

PENNSYLVANIA

Chestnut, D., Education Department, Education Building, Box 911, Harrisburg, PA 17126

Conwell, Marilyn J., 251 W. DeKalb Pike, Apt 909C, King of Prussia, PA 19406

De Cerchio, Donna Eilis, Foreign Language Department, West Chester State College, West Chester, PA 19380

Dugan, Marjorie, Learning Laboratory, Community College-Philadelphia, 34 South 11 th Street, Philadelphia, PA 19107

Eaton, Shirley, 204 West Monument Avenue, Hatboro, PA 19040

Entin, Nathaniel A., Gratz College, 10th Street \& Tabor Road, Philadelphia, PA 19141

Fisher, Ronald D., Language Laboratory, LaSalle College, 20th Street at 01 ney Avenue, Philadelphia, PA 19141

Forquer, Mary Beth, Learning Center, Lehigh University-Coppee Hall 3, Bethlehem, PA 18015

Gennaula, Maryedna Gillespie, 5872 Kings School Road, Bethel Park, PA 15102

Kipphorn, Jr., Richard A., Language Laboratory, Saint Joseph's University, Philadelphia, PA 19131

LaVallee-Williams, Marthe, 1326 Spruce Street, No. 3001, Philadelphia, PA 19107

Lawrason, Robin E., Media Learning Center, Temple University, Humanities B1 dg. HL-21, Philadelphia, PA 19122

Parr, Susan, Language Laboratory, Juniata College, Huntingdon, PA 16652

Pennsylvania State Library, Serial Records Section, Box 1601, Room 46, Harrisburg, PA 17126

Smith, Philip D., Languages, West Chester State College, West Chester, PA 19380

Vernick, Judy A., Language Laboratory, University of Pittsburgh, $732 \mathrm{C}$ of $\mathrm{L}$, Pittsburgh, PA 15260

Warkentin, Henry, Forefgn Language Department, Shippensburg State College, Shippensburg, PA 17257 
West Chester State College, Francis H. Green Library, Department of Education, West Chester, PA 19380

\section{PUERTO RICO}

Inter-American University, Library, San German, PR 00753

\section{RHODE ISLAND}

Language Laboratory Library, Brown University, Box E, Providence, RI 02912

\section{SOUTH CAROLINA}

Arnold, E. P., Dept. of Languages, Clemson University, Clemson, SC 29631

Cherry, Charles Maurice, Modern Language Laboratory, Furman University, Greenville, SC 29613

Galloway, V. , Foreign Language Consultant, Education Department, 801 Rutledge, Columbia SC 29201

SOUTH DAKOTA

Schladweiler, K., Language Arts, Education Division, Kneip Building, Pierre, SD 57501

University of South Dakota, ID Weeks Library, Vermillion, SD 57069

\section{TENNESSEE}

Mc'Connel1, Richard, Dept. of Foreign Languages, Memphis State University, Memphis, TN 38152

Morrison, Robert R., Southern Missionary College, P.0. Box 475, Collegedale, TN 37315

Pleasants, J. V., 2815 Treasure Island East, Memphis, TN 38115

Smith, G., Foreign Language Specialist, Education Language Department, Memphis State University, Memphis, TN 38152

\section{TEXAS}

Allen, Ernest, 1 Allen Farm Road, Fort Worth, TX 76116

Cate, Robert L., Modern Language Laboratory, University of Texas-E1 Paso, El Paso, TX 79968

Edmonds, Victor, Media Services, Our Lady of the Lake University, 411 SW 24 th Street, San Antonio, TX 78285

Gionet, Arthur J., Foreign Lang \& Lit, North Texas State Univ, Denton, TX 76203

McKay, Jym M., Dept. of Speech Communication, University of Texas, Austin, TX 78712

Menin, J. L., Language Laboratory, Rice University, Rayzor Hall 106, Houston, TX 77005

Moses, James 0., Austin State University, Box 13042, Nacogdoches, TX 75962

North Texas State Univ, Acquisitions Services, P.O. Box 5188, Denton, TX 76203

Pierce, James A., Dept. of English, Del Mar College-East, Corpus Christi, TX 78404

Seng, Mark W., University of Texas, EDB 562 FLEC, Austin TX 78741

Sheehan, Joseph H., Language \& Culture Center-English, University of Houston, Houston, TX 77004

Texas Tech University, Germanic \& Slavic Languages, P.0. Box 4579, Lubbock, TX 79409

Van 01phen, H., Language Laboratory, Batts Hall 1, Austin, TX 78712

West Texas State University, Cornette Library, Box 748, W.T. Station, Canyon, TX 79016

UTAH

Burggraaf, Samuel E., Learning Res. Ctr., Brigham Young University, W-162 Stadium, Provo, UT 84602

Howe, E., Foreign Language Education, Education Department, 250 East 500 South, Salt Lake City, UT 84111 
Luckau, Paul F., Germanic Languages, Brigham Young University, 270 MSRD, Provo, UT 84602

VERMONT

Dodge, James W., Secretary Treasurer, Northeast Conference, P. 0. Box 623, Middlebury, VT 05753

St. Michael's College, Attn: Norman Lacharite, Director, International Studies, Winooski, VT 05404

VIRGIN ISLANDS

Language Instruction Supervisor, Education Department, P. 0. Box 630, St. Thomas, VI 00801

VIRGINIA

GERMAN QUARTERLY, Editor, German \& Slavic Languages, University of Virginia, Charlottesvilie, VA 22901

Hi11, Ann, Language Laboratory, Randolph-Macon Woman's College, 2400 Rivermont Avenue, Lynchburg, VA 24503

Instructional Materials Center, 201 East Nine Mile Road, Highland Springs, VA 23075

Reimers, Theresia E., Department of Modern Languages, Hollins College, Hollins College, VA 24020

Thomas, J. E., Learning Laboratory, Virginia Polytechnic Institute, Blacksburg, VA 24061

Virginia Polytechnic Institute, Newman Library-Serials, Blacksburg, VA 24061

Warriner, H., Bilingual Education, $P$. 0 . Box 6Q, Richmond, VA 23216

Watson, John A., Language Laboratory, Virginia Union University, Richmond, VA 23220

Wilde, Karen, 1605 N. Fillmore St., Arlington, VA 22201

\section{WASHINGTON}

Allen, Nina A., Language Learning Center $\mathrm{DH}-40$, University of Washington, Seattle, WA 98195

Augerot, James E., Language Learning Center, University of Washington, Seattle, WA 98195

Bocaz-Moraga, Sergio, Dept. of Modern Languages, Eastern Washington University, 3144 Patterson Hail, Cheney, WA 99004

Crosbie, K., Bilingual Instruction, Public Instruction, 01d Capitol Building, Olymia, WA 98504

Elmendorf, William E., Language Laboratory, Western Washington State College, Bellingham, WA 98225

Johnson, Barbara, Foreign Language Department, Washington State University, Pullman, WA 99164

\section{WISCONSIN}

Bosworth, Lewis A., New Student Services, University of Wisconsin, 432 N. Murray St., Madison, Wi 53706

Grittner, F., Foreign Language Education, Public Instruction, 126 Langdon, Madison, WI 53702

Hoff, Roma, 335 Bartlett Court, Eau Claire, WI 54701

Sampon, Victor, Language Laboratory, Wayl and Academy, Beaver Dam, WI 53916

Saylor, Leslie G., Language Laboratory, University of Wisconsin, P.0. Box 413, Milwaukee, WI 53201

Swanson, Severin A., Language Laboratory, University of Wisconsin, Oshkosh, WI 54091

University of Wisconsin, Library-Serials, 728 State Street, Madison, WI 53706

University of Wisconsin-Milwaukee, Library, Serials Department, P.0. Box 604, Milwaukee, WI 53201 
University of Wisconsin-Parkside, Library-Serials, Wood Road, Kenosha, WI 53141 Zimmerman, Sr. Dorothy M., Fourier Laboratory, Mount Mary College, Mi iwaukee, WI 53222

WYOMING

Soumokil, P., Bilingual Education, Hathaway Building, Cheyene, WY 82002

\section{AUSTRALIA}

Dowling, J. K., Language Laboratory, University of Queensland, St. Lucia, Brisbane 4067 AUSTRALIA

Jordan, Terence C., Language Centre, Griffith University, Nathan, Queensland 4111 AUSTRALIA

McAndrew, Alex, Language Study Center, University of New South Wales, Sydney, NSW 2006 AUSTRALIA

Monash University, Periodicals, Clayton, Vic 3168 AUSTRALIA

National Library of Australia, Prel iminary Processing, (NS 110/72), Canberra, ACT 2600 AUSTRALIA

Reeves, Mrs. Gaynor E., Language Laboratory, University of Newcastle 2308, Newcastle, NSW AUSTRALIA

University of Melbourne, Horwood Language Centre, Medlay Building, West Tower, Parkville, Vic 3052 AUSTRALIA

University of Newcastle, Serials, Auchmuty Library, Newcastle, NSW 2308 AUSTRALIA

University of Queensland, Serials Librarian, Main Library, St. Lucia, Qnsind. 4067 AUSTRALIA

University of Sydney, Serials Division 134881, Fischer Library, Sydney, NSW 2006 AUSTRALIA

\section{BELGIUM}

Appl Linguistics Inst Belgium, Attn: Willy Martin, Blijde Inkomststraat 21, 3000 Leuven BELGIUM

\section{BOLIVIA}

DeVargas, Dolores, Instituto de Idiomas, Carilla 51, Tarija BOLIVIA Jabalquinto, Sergio Rios, Casilla 165, Tarija BOLIVIA

\section{CANADA}

Acadia University, Vaughn Memorial Library, Wolfville, NS BOP 1XO CANADA

Aikens, H. F., Part-Time Studies \& Ext, Dalhousie University, Halifax NS B3H $3 J 5$ CANADA

Aucoin, G. E., 34 Langley Avenue, Dartmouth NS B2W 2 Y6 CANADA

Babin, J. W., French, St. Francis Xavier University, Antigonish, NS B2G 1CO CANADA

Carter, Thomas P., Learning Resource Services, Dalhousie Univ-Killam Library, Halifax, NS B3H 4 H8 CANADA

Ciceran, A. J., Language Laboratory, Brock University-Decew Campus, St. Catharines, ont L2S 3AL CANADA

College of Cape Breton, Library-Sydney Campus, Box 5300, Sydney, NS B1P 6L2 CANADA

Concordia University, Library, Serials Acquisitions, Sir George Williams Campus, 1455 Maisonneuve Blvd., West, Montreal PQ H3G 1 M8 CANADA

Couroux, Gerard, College Marie Victorin, 7000 rue Marie Victorin, Montreal, PQ 462 CANADA

Hamm, C., Language Laboratories, Queen's University, Kingston, Ont K7L 3N6 CANADÁ 
Hammerly, Hector, Literature \& Linguistics, Simon Fraser University, Burnaby, BC V5A 1 S6 CANADA

Hennig, C. W., Language Laboratory, University of Waterloo, Room 110, ML Building, Waterloo, ont N2L 3G1 CANADA

Hewson, J., Linguistics, Memorial University of Newfoundland, St. John's, Nfind CANADA

James, E. F., Language Lab, Rm. 259B, University College-University of Toronto, 15 King's College Circle, Toronto, Ont. MSS IAl CANADA

Kenner, R., Language Laboratory-A/N Dept., Concordia University H-399, 1455 de Maisonneuve BIvd., Montreal, PQ H3G IMB CANADA

Kozoriz, G., Canadian Forces Lang School-0tt., National Defense Headquarters, Ottawa, Ont K1A OK2 CANADA

Lemyze, J.-C., Language Laboratory, McGill University, Montreal, PQ H3A $1 \times 9$ CANADA

Majhanovich, Suzanne E., Faculty of Education, University of Windsor, Windsor, Ont, N9B 3P4 CANADA

Marxheimer, Edward, Romance Languages, University of Alberta, Edmonton, Alta T6G 2E6 CANADA

MCGill University Libraries, Serials Department, 3459 McTavish Street, Montreal, PQ H3A 1 HI CANADA

Ministry-Culture \& Recreation, Citizenship Language Classes, 454 University Avenue, Toronto, Ont M4G 1 Y7 CANADA

Ministry-Culture \& Recreation, Resource Centre, 77 Bloor Street West, Toronto, Ont M7A 2R9 CANADA

National Library of Canada, Order Section, 395 Wellington Street, Ottawa, Ont KIA ON4 CANADA

Petherbridge, $D$. L., University of Lethbridge, Lethbridge, Al ta T1K 3M4 CANADA

Pritchard, C., SONY of Canada Ltd, 411 Gordon Baker Rd, Willowdale, ONT MRH 2S6 CANADA

Raymond, Walter, Atelier de langues, CEGEP Ste-Foy, 2410, Chemin Ste-Foy, Ste-Foy, Quebec GIV 1 T3 CANADA

Sawyer, Isabe1, Language, Linguistics \& Literature, Simon Fraser University, Burnaby BC CANADA

Seidel, J., Language Laboratory, University of Victoria, P. 0. Box 1700, Victoria BC V8W 2 Y2 CANADA

Simon Fraser University, Library-Acquisition Division, Burnaby BC V5A 156 CANADA

Thibault, Claude, Notre-Dame-de-Foy, Cap Rouge, PQ GOA 1KO CANADA

University of Alberta, Attn: C. Dechaine, Language Lab, Edmonton, Alta T6G 2E1 CANADA

University of Western Ontario, Periodicals-General Library, London, Ont N6A 3K7 CANADA

Vijayakumar, E. K., Thurber Comprehensive High School, 15 Orilla Park, Red Deer, Alta T4N 5 A6 CANADA

York University Libraries, Central Serials Records, 4700 Keele Street, Downsview, Ont M3J 2R2 CANADA

\section{CHILE}

Baltra, Liliana, CPEIP, Casilla 16162, Santiago 9 CHILE

Universidad de Concepcion, Biblioteca Central, Casilla 1807, Concepcion CHILE

DENMARK

Odense Universitetsbibl, TSS. Campusvej SS, DK-5230 Odense M DENMARK

ECUADOR

Lopez, Jose A., ESPOL, Apartado 5863, Guayaquil 303733 ECUADOR 


\section{ENGLAND}

AV LANGUAGE JOURNAL, Attn: Editor, University of Aston-Birmingham, Gosta Green, Birmingham B4 7ET ENGLAND

AV LANGUAGE JOURNAL, 7 Shelley Close, Langley, Bucks SL3 8JW ENGLAND

British Library, Accessions, Lending Div., Boston Spa, Wetherby, Yorkshire LS23 $7 B Q$ ENGLAND

Ctr Info Lang Teaching, Deputy Librarian, 20 Carlton House Terrace, London SW IY 5AP ENGLAND

\section{FINLAND}

Helsingen Yliopisto, Kielikeskus, Fabianinkatu 26, SF-00100 Helsinki 10 FINLAND Jyvaskyla University, Language Laboratory, SF-40/100 Jyvaskyla 10 FINLAND

Korkeakoulun, Joensuun, Kirjasto, PL111, 80101 Joensuu 10 FINLAND

University of Turku, Language Centre, 20500 Turku 50 FINLAND

\section{FRANCE}

Universite de Strasbourg, Inst Linguistque Appliquee, Laboratoires de Langues, 22, rue Descartes, Strasbourg-Cedex 67084 FRANCE

HUNGARY

Konyvtar, Egyetemi, POB 483, Budapest 1372 HUNGARY

INDIA

Annamalai, E., Central Inst. Indian Lang, Manasagangotri, Mysore-570 006 INDIA ISRAEL

David Yellin Teacher's Sem, Beth-Hakerem, Jerusalem ISRAEL

Jewish National \& University Library, Periodicals, P.0. Box 503, Jerusalem ISRAEL

\section{JAPAN}

Achiba, Machiko, 8-34 Konuinshukusha, 11-72 Sakashita, Okazaki, Aichi-ken 444 JAPAN

Amano, Kazuo, 16-6 Shiomigaoka-Cho, Chiba-Shi 280 JAPAN

Cox, Ronald M., Engl ish Program-Personnel, Kobe Steel, Ltd., 3-18, 1-chome, Wak inohama-cho, Chuo-ku, Kobe 651 JAPAN

Fukuoka University, LL Room, Nanakuma, Fukuoka 814 (MZ) JAPAN

Gifu Jyoshi Tanki Daigaku, 2693 Fukumi tsu, Nagara, Gifu 502 MZ JAPAN

Hanzel, Louis F., Nanzan University, 18 Yamazato-cho, Showa-ku, Nagoya 466 JAPAN

Hiroshima Daigaku, Honbu Toshokan-K, Higashisendamachi, Hiroshima 730 MZ JAPAN

Hiroshima Shudo Daigaku Lib, Otsuka Numata-Cho, Hiroshima (Kin) 731-31 JAPAN

Honda, Ritsuri, Assumption College, 2-16 0te-cho, Takatsuki-shi, Osaka 569 JAPAN

Ikeura, Sadahiko, Fukuoka University of Education, 279 Akama Munakata-gun, FukUoka JAPAN

Kagawa University, Library, Saiwai-Cho, Takamatsu-Shi 760 (Kin) JAPAN

Kanagawa University, Library, Rokkakubashi, Kanagawa-Ku, Yokohama (MZ) 221 JAPAN

Kansai University Library, P. 0. Box 50, Suita, Osaka $564 \mathrm{MZ}$ JAPAN

Kitao, Kenji, Dept. of English, Doshisha University, Kariigyo-ku,, Kyoto 602 JAPAN 
Kogyo Kure Koto, Senmon Gakko Lib, Aga-Minami-2-2-11, Kure 737 MZ JAPAN

Kohmoto, Sutesaburo, 4-16-17 Meguro, Meguro-ku, Tokyo 153 JAPAN

Kurita, Katsumi, SONY Language Laboratory, Hiyama B1 dg, \#2, 4F1, 2-20-30, Takanawa, Minato-Ku, Tokyo 108 JAPAN

Kuroda, Takashi, Engl ish Literature, Otsuma Womens College, 12, 3-bancho, Chiyoda-ku, Tokyo 102 JAPAN

Kyoto Sangyo Daigaku, Toshokan, (Kyoyo-Eigo-Ken), Kita-Ku Kyoto 603 MZ JAPAN

Kyushu Sangyo Daigaku, (LL Room), 327, Matugadai-2-chome, Hi gashi-ku, Fukuoka City 813 MZ JAPAN

Lance, John, Japan-Amer Conversation Inst., Intl. Education Center, 21 Yotsuya, 1-chome, Shinjuku-17, Tokyo 160 JAPAN

Miura, Shogo, 72-121, Jike, Sayo-cho, Higashi-Hiroshima 724 JAPAN

Nagoya Gakuin Daigaku, Gaikokugo Center, Kami shinano-Cho, Seto, Aichiken 480-12, MZ JAPAN

O'Donohue, Barbara, Hiroshima Bunkyo Womens College, Kabe Cho-Asa Kita Kucho, Hiroshima 731-92 JAPAN

Peterson, Greg, Notre Dame Women's College, Shimogamo, Sakyo-ku, Kyoto 606 JAPAN

Rawlings, Ruth, 101 Kobuke Cho, Chiba Shi, Chiba Ken 281 JAPAN

Saga University, Honjyo-Machi, Saga City 840 MZ JAPAN

Sapporo Ika Daigaku, Toshokan S-1 W-17, Sapporo 060 (KIN) JAPAN

Seinan Gakuin University, Language Lab, 6-2-92 Nishijin Nishi-Ku, Fukuoka 814 JAPAN

Tokyo-Seitoku-Tanki-Daigaku, Library (MZ), 7-13, Jujodai-1-Chome, Kita-Ku, Tokyo JAPAN

\section{NETHERLANDS}

Rijksunivers Te Groningen, Bibliotheek, Postbus 559, Oude Kijk In't Jatstraat 5 , Groningen NETHERLANDS

Universiteitsbibliotheek 1439, A. 324. 639, Eras Muslaan 36, Nijmegen 1 NETHERLANDS

\section{NEW ZEALAND}

Norrish, N., Language Laboratory, University of Victoria, Private Bag, Hellington NEW ZEALAND

\section{NORWAY}

Moseby, Erik, Tandberg A/S, P. O. Box 55 - Bogerud, Os lo 6 NORWAY

\section{SAUDI ARABIA}

Lewis, Steven F., GEP Training, Lockheed Aircraft International, A.G., Riyadh SAUDI ARABIA

\section{SCOTLAND}

University of St. Andrews, Attn: Ms. Konn, Modern Language Library, Buchannon Bldg - Union Street, St. Andrews, Fife KY16 9PH SCOTLAND

\section{SINGAPORE}

National University Singapore, Kent Ridge Library, Chief Librarian, Kent Ridge SINGAPORE

SEAMEO Reg. Language Center, Librarian of Information Center, 30 Orange Grove Road, Singapore 1025 SINGAPORE 
University of South Africa-Port Elizabeth, Library-Periodicals Dept., Private Bag 6058, Port Elizabeth 6000 SOUTH AFRICA

University of Stellenbosch, Library, Private Bag 5036, Stellenbosch 7600 SOUTH AFRICA

\section{SWEDEN}

Davies, Norman, University of Linkoping, S-581 83 Linkoping SWEDEN

\section{SHI TZERLAND}

Bennet, T.J.A., Language Laboratories, Hochscule St. Gallen, Dufourstrasse 50, St. Gailen, 9014 SWITZERLAND

Universitat Bern, Verwaltung, Hochschul strasse 4, 3012 Bern SWITZERLAND

Universite de Neuchatel, Chaire de Linguistique Generale, Neuchatel, 2000 SWITZERLAND

\section{THAILAND}

Sananvanit, Ms. Kwanchai, SEA Enterprises Co., Ltd., Paris Theatre, 292/15-16 Luk Luang Road, Bangkok 3 THAILAND

USSR

Krupatkin, Yakov Borisovich, u.1. Odesskaja 1, kv. 19, Sevastopol 335000 USSR

\section{WEST GERMANY}

Buchandlung Lehmkuh1, Leopoldstrasse 45, 8000 Munchen 40 WEST GERMANY

Free University of Berlin, Foreign Language Laboratories, Habelschwerdter Allee 45, Ihnenstrasse 24, 1 Berlin 33 (Dahlem) WEST GERMANY

Freudenstein, Reinhold, Foreign Language Research Info. Ctr., Lahnberge, D-3550 Marburg/Lahn WEST GERMANY

Stadt \& Universitatsbibi, Bockenheimer Landstr 134, 6000 Frankfurt Am Main WEST GERMANY

Universitatsbibliothek, Zeitschriften-FGSZ, Universitatsstrasse 4, 8520 Erlangen WEST GERMANY

\section{ZIMBABWE}

University of Zimbabwe, Language Laboratory \#3643, P. 0. Box MP 167, Mount Pleasant, Salisbury ZIMBABWE 